\title{
Cigarette smoking and body weight: information may be hazardous to your health
}

As scientists, academicians, and educators, it is clear that we should provide complete and accurate information about everything that we learn. As healthcare practitioners, however, we are in somewhat of a dilemma when correct information is likely to result in unhealthy behaviours. This dilemma is more than an exercise in ethics to debate in medical and graduate schools; it is a reality that we face today with regard to tobacco use and body weight.

In the late $1970 \mathrm{~s}$, I became interested in tobacco use and body weight for several reasons. First, my mentor (Stanley Schachter) had studied each of the topics separately and I hoped to integrate the work. ${ }^{12}$ Second, I hoped to figure out a new way to study the underlying biobehavioural bases of drug addiction and I believed that studying tobacco use, nicotine, eating, and body weight might provide valuable insights. Third, I decided that even if my grand goals could not be reached, at least I would study two important health-related conditions. The information that we gathered about smoking, nicotine, and body weight in many experiments did, indeed, move knowledge forward. ${ }^{3}$ I did not consider, however, that information that I and other investigators reported about tobacco and body weight would contribute to tobacco use and abuse.

A thorough review of the tobacco literature reveals that it has long been assumed that tobacco use is inversely related to body weight. This knowledge anecdotally dates back millennia and scientifically dates back more than 100 years. However, careful scientific investigation of this relationship did not emerge until the 1980s. Then, again and again it became clear that what our grandparents knew to be true is indeed true: tobacco use helps control body weight for many people. Scientific reports built on this information in important ways by identifying nicotine, the primary drug of addiction in tobacco, as the primary cause of body weight effects. Further, it has become clear that nicotine alters energy intake of specific foodstuffs as well as energy expenditure. ${ }^{3}$

This information has fallen into that group of scientific discoveries that health journalists find readily accessible and relevant. Therefore, newspapers, magazines, and other media have been filled with reports that tobacco helps to control body weight.

Clear findings on this topic also have resulted in many investigations in the laboratory with humans and animals, in field settings, and in clinics. These investigations provide additional, useful information that may help people avoid weight gains after smoking cessation, that may help us understand why people smoke, and that may help us understand underlying biobehavioural mechanisms of addiction. Unfortunately, this information also may encourage some people to start smoking.

The article by Klesges et $a l^{4}$ in this issue of Tobacco Control provides further evidence that many adolescents are aware of the inverse relationship between tobacco use and body weight and that many adolescents purposely smoke cigarettes to help control their body weight. These investi gators report that Caucasian girls are especially concerne about body weight and are most likely to smoke because of body weight. This finding corroborates other reports and consistent with general beliefs and stereotypes? Additionally, Klesges et al report that extremely few African-American boys and girls smoke. This finding als $\vec{\theta}$ is consistent with other reports and remains an important finding that deserves detailed examination to find out whow is preventing smoking initiation in African-American chifo dren.

The fact that many Caucasian girls choose to smoke because of body weight, despite the well-publicised hazards of tobacco use, is an indication of the serious pres? sures on such girls to achieve and maintain low body weight. That African-American girls are markedly less likely to start smoking may reflect differences in perceived. ideal body weight between the races. It is truly disturbing that images of overly thin female role models, particularly Caucasian models and performers, permeate American culture. It is interesting and worth investigating wh African-American girls and boys are not choosing t smoke. Perhaps Aretha Franklin continues to be a role् model for young African-American people, whereas Alanis Morissette is a role model for young Caucasian people This possible difference may help explain racial differences in smoking by girls, but it still does not explain why there such a marked difference in smoking by boys of these two races.

It is clear that there is much work to do to help preven smoking. It is less clear, however, how we should best com municate information that we acquire, such as the fact that smoking helps control body weight. Providing completê and accurate information certainly is a goal that we attempt to achieve, but it is sobering to consider that infor: mation that we provide may sometimes be damaging tô some people's health.

Medical and Clinical Psychology,

Uniformed Services University of the Health Sciences, Bethesda, Maryland 20814-4799, USA

email:grunberg@usuhsb.usuhs.mil

NEIL E GRUNBER

1 Schachter S, Rodin J. Obese humans and rats. Potomac, Maryland: Lawrence Erlbaum Associates, 1974.

2 Schachter S, Silverstein B, Kozlowski LT, Perlick D, Herman CP, Lieblin B. Studies of the interaction of psychological and pharmacological determinants of smoking. ₹ Exp Psychol Gen 1977;106:3-40.

3 Grunberg NE. Cigarette smoking and body weight: A personal journe through a complex field. Health Psychol 1992;11 Suppl:26-31.

4 Klesges RC, Elliott VE, Robinson LA. Chronic dieting and the belief that smoking controls body weight in a biracial, population-based adolescen sample. Tobacco Control 1997;6:89-94.

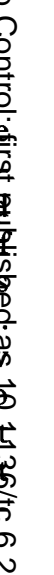

